Short Research Communication

\title{
Involvement of necroptosis, a newly recognized cell death type, in steroid-induced osteonecrosis in a rabbit model
}

\author{
Toru Ichiseki $\mathrm{i}^{1^{*}}$, Shusuke Ueda ${ }^{*}$, Yoshimichi Ueda ${ }^{2}$, Masanobu Tuchiya ${ }^{1}$, Ayumi Kaneuji ${ }^{1}$ and Norio \\ Kawahara ${ }^{1}$ \\ 1. Department of Orthopaedic Surgery, Kanazawa Medical University, Daigaku 1-1, Uchinada, Kahoku-gun, Ishikawa, 920-0293, Japan; \\ 2. Department of Pathology, Kanazawa Medical University, Daigaku 1-1, Uchinada, Kahoku-gun, Ishikawa, 920-0293, Japan. \\ * These authors contributed equally to this work. \\ $\triangle$ Corresponding author: Toru Ichiseki M. D., PhD., Department of Orthopaedic Surgery, Kanazawa Medical University, Daigaku 1-1, Uchinada-machi, \\ Kahoku-gun, Ishikawa 920-0293, Japan. Phone: +81-76-286-2211, ext. 3214, Fax: +81-76-286-4406; Email: tsy-ichi@kanazawa-med.ac.jp. \\ (C) Ivyspring International Publisher. This is an open access article distributed under the terms of the Creative Commons Attribution (CC BY-NC) license
} (https://creativecommons.org/licenses/by-nc/4.0/). See http://ivyspring.com/terms for full terms and conditions.

Received: 2016.08.08; Accepted: 2016.11.24; Published: 2017.01.25

\begin{abstract}
We investigated the role of programmed necrosis (necroptosis), a newly recognized form of cell necrosis that has been implicated in the development of steroid-induced osteonecrosis. We used an osteonecrosis model in which 30 Japanese white rabbits each weighing $3.5 \mathrm{~kg}$ were injected once with methylprednisolone at $20 \mathrm{mg} / \mathrm{kg}$ body weight into the right gluteal muscle. Ten animals killed 14 days thereafter were designated as S14d groups, while another 10 animals injected with necroptosis, a specific inhibitor of necrostatin-1 i.v. at $1.65 \mathrm{mg} / \mathrm{kg}$ on the same day as the steroid were also killed on the $14^{\text {th }}$ day and designated as SN14d group. As a control, 10 animals injected only with physiological saline were studied as $\mathrm{N}$ group. After the animals were sacrificed the bilateral femoral bone was examined histopathologically and the presence of osteonecrosis determined. Furthermore, animals subjected to the same treatment and killed on the $3^{\text {rd }}$ day after drug administration were set up as S3d group and SN3d group, and Western blotting of Receptor-interacting protein (RIP) 1 and RIP3 in femoral bone performed. The osteonecrosis rate was $70 \%$ in S14d group, and $0 \%$ in both N and SN groups. In 2 of 10 animals in SN group fatty marrow was found. On Western blotting significantly increased expression of both RIPI and RIP3 was noted in S3d group, confirming that Nec-1 was suppressed. Necroptosis mediated by RIPI and RIP3 expression was thought to be implicated in the development of steroid-induced osteonecrosis. Also, by suppressing expression of RIPI and 3 with the administration of Nec-1 the osteonecrosis rate was significantly decreased. These results suggest that necroptosis may have potential as a novel target for both elucidating the mechanisms underlying steroid-induced osteonecrosis and establishing more effective prophylactic countermeasures.
\end{abstract}

Key words: steroid-induced osteonecrosis, necroptosis, receptor-interacting protein (RIP), necrostatin-1 (nec-1).

\section{Introduction}

The developmental mechanisms of steroidinduced osteonecrosis of the femoral head have been investigated from a variety of aspects, and thus far factors such as steroid-induced coagulation/ fibrinolytic system and lipid metabolism abnormalities, blood flow disturbances, and oxidative stress have all been implicated [1-3]. Moreover, as preventative approaches the administration of lipid metabolism enhancing agents having antioxidant effects or of antioxidants such as vitamin $\mathrm{E}$ and reduced-Glutathione has been demonstrated to achieve a significant inhibitory effect [4-7]. However, much still remains obscure about the developmental mechanisms of osteonecrosis even at the present time. 
One reason for this is that since, in general, necrosis is a non-programmed type of cell death analysis of its molecular mechanisms has not proceeded very far. The situation regarding the mechanisms underlying steroid-induced osteonecrosis is similar in that despite the extensive research focused on this subject many points remain unclear. For this reason additional investigations from diverse viewpoints are still very much needed.

Meanwhile, various studies have recently implicated necroptosis in the pathogenesis of necrotic lesions, and its importance in this context has been increasingly recognized. Necroptosis is a new concept of cell death recently proposed to help explain necrosis that suddenly becomes uncontrollable $[8,9]$. Necroptosis is a programmed necrosis, and a signal of cell death that has been found to be involved in various necrosis-related disorders including cerebral infarction, cardiac disease, and some intestinal disorders. At present, necroptosis is also recognized to play a role in various other contexts such as ischemia-reperfusion injury, elimination of various kinds of viral infection, onset of drug-induced pancreatitis, and diseases induced by ischemiahypoxia [10].

Necroptosis has also been said to influence the role played by receptor-interacting protein (RIP) 1 and RIP3 in cell death, and to play roles in disease development and progression [11]. On the other hand, necroptosis has been shown to be suppressible by the RIP1 inhibitor necrostatin-1 (Nec-1) [12].

Since steroid-induced osteonecrosis is also considered to be due to ischemia-hypoxia, it can easily be conjectured that necroptosis may similarly play a role in its development. Also, in the present model the process leading to osteonecrosis has been reported to begin as early as 1-3 days after steroid administration, with osteonecrosis seen to develop in about $60-70 \%$ of animals by 14 days after steroid administration $[13,14]$.

The rabbit model used here has been widely used as a model of steroid-induced osteonecrosis and was deemed suitable to investigate RIP1 and RIP3 expression in the early period after steroid administration as well as the inhibitory effect on osteonecrosis of administration of Nec-1, an RIP1 inhibitor. In this way we hoped to define the role played by necroptosis in steroid-induced osteonecrosis.

\section{Materials and methods}

\section{Animals}

1) Thirty adult female Japanese white rabbits (mean body weight $3.5 \mathrm{~kg}$ ) were injected once with methylprednisolone at $20 \mathrm{mg} / \mathrm{kg}$ body weight into the right gluteal muscle, and 10 animals were killed 14 days after steroid administration and designated as group S14d. Another 10 animals injected i.v. with necroptosis, a specific inhibitor of Nec-1 at $1.65 \mathrm{mg} / \mathrm{kg}$ on the same day as the steroid were also killed on the $14^{\text {th }}$ day and designated as SN14d group. As a control (N group), 10 animals administered only physiological saline as a vehicle injection were also compared.

Immediately after the animals were killed the bilateral femurs were isolated and fixed in 10\% formalin for 1 week, and the specimens were decalcified in 10\% EDTA. The specimens were then embedded in paraffin, and cut into $4 \mathrm{~mm}$ sections.

2) Having noticed that the process leading to osteonecrosis develops within 3 days of steroid administration, on day 3 after steroid administration we used Western blot method to study the expression of RIP1 and RIP3 (S3d group, $\mathrm{n}=5$ ). In addition, like in experiment 1, Nec-1 was injected i.v. at $1.65 \mathrm{mg} / \mathrm{kg}$ on the same day as the steroid, and on day 3 bilateral femoral head bone was harvested from 5 animals, which were designated as SN3d group. As a control, 5 animals injected only with physiological saline were compared as $\mathrm{N}$ group. Each group consisted of 5 animals, and bilateral femoral head bone was cryopreserved at $-80^{\circ} \mathrm{C}$ after resection. All protocols in this study were performed in accordance with the guidelines of the Animal Research Committee of Kanazawa Medical University.

\section{Histopathology}

Specimens prepared in 1) were used. Necrosis of bone and marrow tissues was examined in hematoxylin-eosin-stained preparations by light microscopy. Osteonecrosis was judged to be present when necrosis of medullary hematopoietic cells or fat cells or empty lacunae or condensed nuclei in osteocytes were noted. Osteonecrosis was judged to be present when osteonecrosis was identified in either isolated femur [9]. The rate of development of osteonecrosis was calculated as the ratio of the number of rabbits with osteonecrosis to the total number of rabbits used.

\section{Western blotting}

Samples prepared in 2) were used. Immunoblotting for RIP1 and RIP3 was performed on the proximal femur of steroid administered rabbits, as well as non-treated rabbits. Proteins were extracted using lysis buffer $(50 \mathrm{mM}$ Tris- $\mathrm{HCl}, \mathrm{pH}$ 7.6, 10\% glycerol, $5 \mathrm{mM}$ magnesium acetate, $0.2 \mathrm{mM}$ ethylenediamine tetraacetic acid, $1 \mathrm{mM}$ phenylmethylsulfonyl fluoride, and $1 \%$ sodium 
dodecylsulfate). Extracted protein (20 mg) was applied to and electrophoresed on a $10 \%$ polyacrylamide gel, and transferred to a nitrocellulose membrane (Atoh, Tokyo, Japan). The membranes were reacted overnight at $4^{\circ} \mathrm{C}$ with anti-RIP1 polyclonal antibody and anti-RIP3 polyclonal antibody at a concentration of 1:200 dilution and 1:100 dilution respectively. After incubation with peroxidase-labeled goat anti-rabbit IgG antibody (Dako Cytomation) for 1 hour at room temperature and vigorous washing, the nitrocellulose membrane was incubated with ECL-pulse (GE Medical, USA) and photographed digitally using LAS4000 (FUJIFILM, Tokyo, Japan). All samples were standardized by immunoblot using anti- $\beta$ actin mouse monoclonal antibody (Sigma Chemical Co., St. Louis, MO).

\section{Quantification of the Western blot results}

Protein quantification was done using analytical software "Multi Gauge" (FUJIFILM, Tokyo, Japan). $\beta$-actin was used as the control for all specimens in each group. All values were expressed as the mean \pm $\mathrm{SD}$, and Arbitrary Units (AU) were used at the time of measurement.

\section{Statistical analysis}

RIP1 and RIP3 levels were expressed as the mean \pm SD. Statistical analysis was performed using analysis of variance with Dunnett's multiple comparison test. By using this test we were able to determine the point in time after administration of steroid that RIP1 and RIP3 levels showed any significant change, as compared with the values in $\mathrm{N}$ group. The rate of ON development was compared between S14d and SN groups using Yates's modified chi-squared test. $P$ values less than 0.05 were considered significant. Statistical analysis was performed using StatView J-5.0 software (SAS Institute).

\section{Results}

\section{Histopathology}

Osteonecrosis was not found in any of the animals of $\mathrm{N}$ group. In S14d group, osteonecrosis was found in 7 of 10 animals $(70 \%)$, consistent with previously reported results. Necrotic areas in group S14d were clearly demarcated from the surrounding normal tissue. Empty lacunae were found in bone trabeculae, and the surrounding bone marrow tissue also showed necrotic changes (hematopoietic cell and fat cell necrosis). On the other hand, in SN group, osteonecrosis was not found in any of the animals for an osteonecrosis rate of $0 \%$ and representing a significant decrease as compared to S14d group $(p<0.05)$. However, findings suggestive of steroidinduced fatty marrow were noted in 2 animals. (Figure 1)
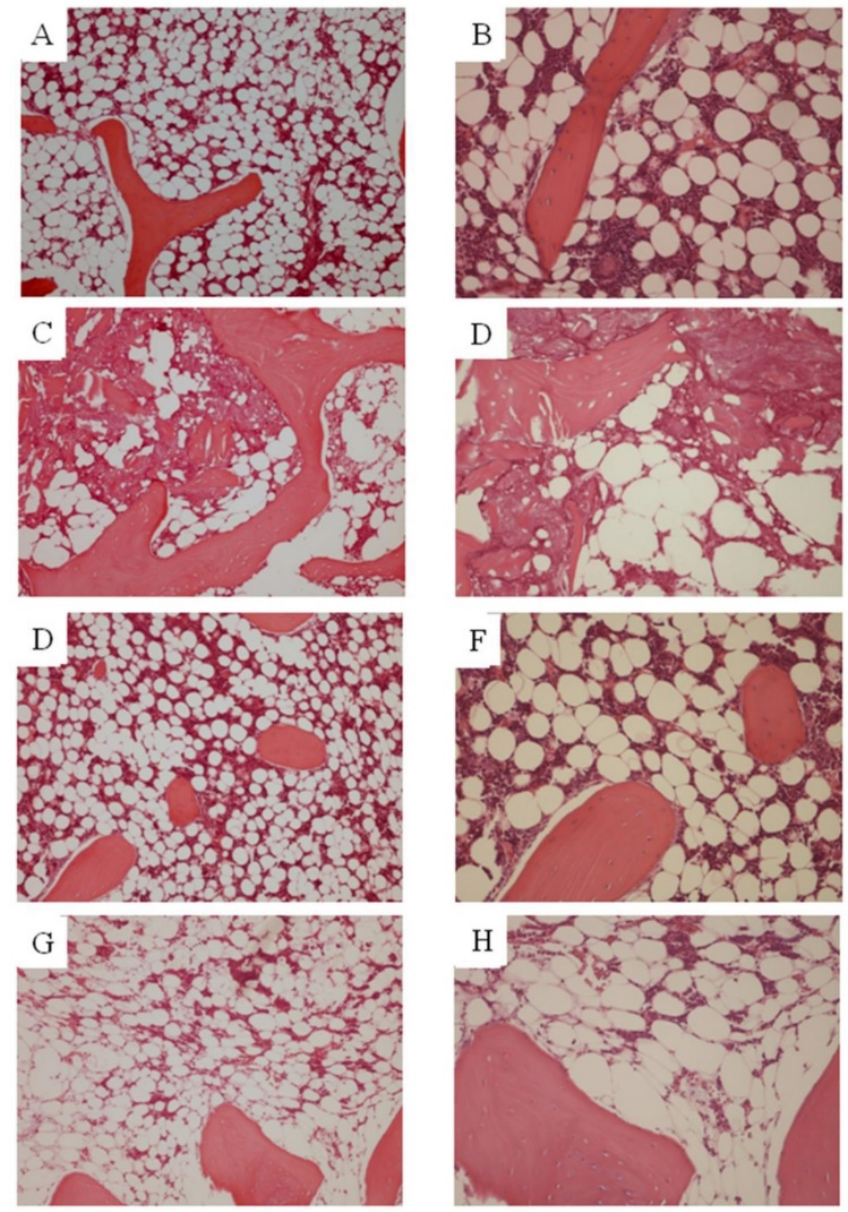

Figure 1. Histopathological study. A, B. Ngroup. C, D. S14d group. E, F. SN group (osteonecrosis is absent). G, H. SN group (fatty marrow) (A,C,E,G. $\times 100)(B, D, F, H . \times 200)$. Osteonecrosis was not found in any of the animals in either $\mathrm{N}$ or SN group. In St 14d group, osteonecrosis was found in 7 of animals (70\%). Osteocytes in the stained bone contain achromatic nuclei or empty lacunae, showing typical features of osteonecrosis. Medullary hematopoietic cells around the site of osteonecrosis are interspersed with necrotic and degenerated cells. In SN group, some degree of fatty marrow was found in 2 of 10 animals.

\section{Western blot for RIPI and RIP3}

Approximate molecular weights of around 76 $\mathrm{kDa}$ for RIP1 and $57 \mathrm{kDa}$ for RIP3 were demonstrated by western blot analysis. In S3d group as compared to $\mathrm{N}$ group increased expression of both RIP1 and RIP3 was found. On the other hand, in SN3d group which had received Nec-1, expression of both RIP1 and RIP3 was confirmed to be inhibited as compared with S14d group. Next, quantification of RIP1 and RIP3 expression by Western blot was compared using analytical software. In N group RIP1 was $(6.4 \pm 0.43)$ $\mathrm{AU}$ in contrast to $(12.8 \pm 0.66) \mathrm{AU}$ in $\mathrm{S} 3 \mathrm{~d}$ group $(\mathrm{P}<0.001)$. Also, in SN3d group (6.7 \pm 0.61$)$ AU to which Nec-1 had been administered a significant decrease 
was found as compared to S3d group, whereas the difference with $\mathrm{N}$ group was not significant. Similarly for RIP3, a significant increase was found in S3d group (13.8 \pm 0.67 ) $\mathrm{AU}$ as compared to $\mathrm{N}$ group $(2.9 \pm 0.13)$ AU $(p<0.001)$. A significant inhibition of expression was found in $\mathrm{SN}$ at $(3.4 \pm 0.31) \mathrm{AU}$, in the comparison with S3d group, whereas the difference with $\mathrm{N}$ group was not significant (Figure 2).

\section{Discussion}

The regulation of cell death is extremely important both physiologically and pathologically, and is in general classified into apoptosis and necrosis. Apoptosis is a kind of programmed cell death implemented by a mechanism in which caspase, etc. are genetically inhibited and physiological mechanisms such as morphogenesis in the developmental process and removal of virally infected cells are involved [15]. On the other hand, hitherto the molecular mechanisms of necrosis have not been extensively analyzed despite its purported importance in various pathological states. One reason for this is that necrosis has been classified as an incidental type of non-programmed cell death induced by factors such as excessive physical stress. However, recently, analyses of cell death mediated by Death receptors such as FAS have been conducted, and the existence of a programmed type of necrosis. called necroptosis, has been reported $[16,17]$.

Hitherto diseases associated with necrosis have been considered difficult to control because of the non-programmed nature of the necrosis. But more recently, necroptosis has been increasingly implicated in this kind of disease, and RIP1 has been found to be essential for the establishment of necroptosis [18].
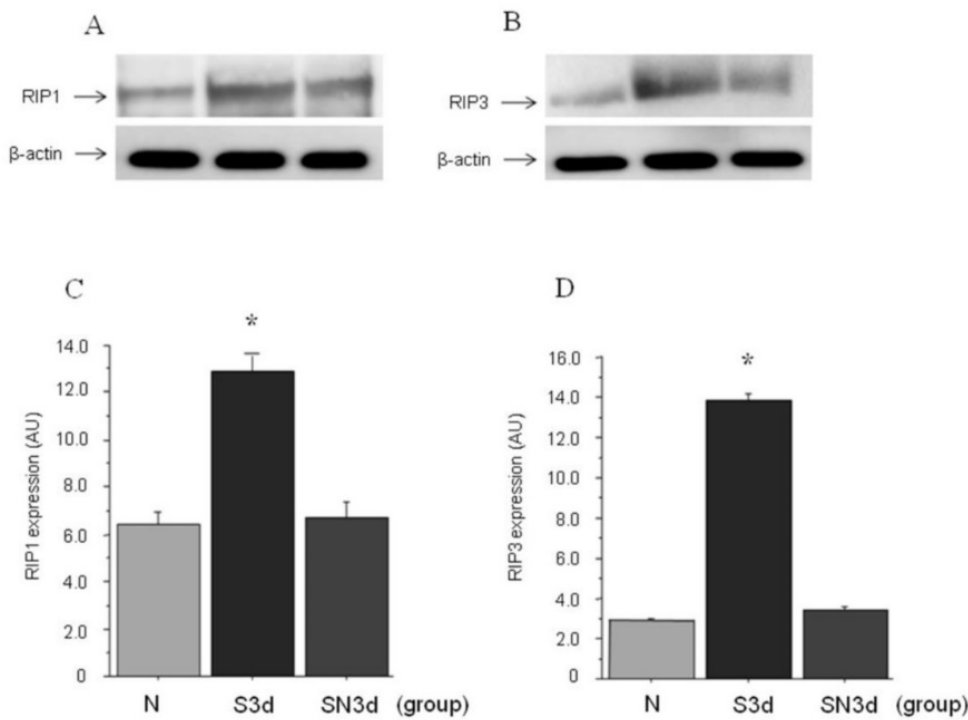

Figure 2. Western blot study. A. expression of RIP1; B. expression RIP3. C, D. quantification of RIP1 and RIP3 expression by Western blot. RIP1 induced significantly increased expression in S3d group, while with Nec-1 administration it was significantly inhibited to the same degree as N group. RIP3 like RIPI showed significantly increased expression in S3d group, while with $\mathrm{Nec}-1$ administration it was significantly inhibited to the same degree as $\mathrm{N}$ group.(*: $\mathrm{p}<0.001)$ 
However, as is well known, steroid use is associated with the development of porosis. In the present study too porosis-like changes in bone marrow were found in 2 animals.

If considering inhibition of bone marrow changes such as porosis as well, it may also be necessary to determine the optimal dosing and number of administrations of $\mathrm{Nec}-1$.

The results of this study showed that after steroid administration an increase in the expression of RIP1and RIP3 was found in femoral head bone, and that the administration of necrostatin significantly inhibited the expression of RIP1 and RIP3 and development of osteonecrosis. These results suggest that RIP1 and RIP3 may play an important part in steroid-induced osteonecrosis development. For this reason, necroptosis may represent a novel target for elucidating the mechanisms underlying steroid-induced osteonecrosis and suggesting new preventative approaches.

\section{Competing Interests}

The authors have declared that no competing interest exists.

\section{References}

[1] Mankin HJ. Nontraumatic necrosisof bone (osteonecrosis). N Engl J Med 1992; 326: $1473-1479$.

[2] Motomura G, Yamamoto T, Miyanishi K, Jingushi S and Iwamoto $Y$. Combined effects of an anticoagulant and a lipid-lowering agent on the prevention of steroid-induced osteonecrosis in rabbits. Arthritis Rheum 2004; 50: 3387-3391.

[3] Ichiseki T, Kaneuji A, Ueda Y, Nakagawa S, Mikami T, Fukui K and Matsumoto T. Osteonecrosis development in a novel rat model characterized by a single application of oxidative stress. Arthritis Rheum 2011; 63: 2138-2141.

[4] Mikami T, Ichiseki T, Kaneuji A, Ueda Y, Sugimori T, Fukui K and Matsumoto T. Prevention of steroid-induced osteonecrosis by intravenous administration of vitamin E in a rabbit model. J Orthop Sci 2010; 15: 674-677.

[5] Ichiseki T, Matsumoto T, Nishino M, Kaneuji A and Katsuda S. Oxidative stress and vascular permeability in steroid-induced osteonecrosis model. J Orthop Sci 2004; 9: 509-515.

[6] Kuribayashi M, Fujioka M, Takahashi KA, Arai Y, Ishida M, Goto T and Kubo T. Vitamin E prevents steroid-induced osteonecrosis in rabbits. Acta Orthop 2010; 81: 154-160

[7] Nishida K, Yamamoto T, Motomura G, Jingushi S and Iwamoto Y. Pitavastatin may reduce risk of steroid-induced osteonecrosis in rabbits: a preliminary histological study. Clin Orthop Relat Res 2008; 466: 1054-1058.

[8] Alexei Degterev, Junichi Hitomi, Megan Germscheid, Irene L Ch'en, Olga Korkina, Xin Teng, Derek Abbott, Gregory D Cuny, Chengye Yuan, Gerhard Wagner, Stephen M Hedrick, Scott A Gerber, Alexey Lugovskoy and Junying Yuan. Identification of RIP1 kinase as a specific cellular target of necrostatins. Nat Chem Biol 2008; 4: 313-321.

[9] Junichi Hitomi, Dana E Christofferson, Aylwin Ng, Jianhua Yao, Alexei Degterev, Ramnik J Xavier and Junying Yuan. Identification of a molecular signaling network that regulates a cellular necrotic cell death pathway. Cell 2008; 135: 1311-1323.

[10] Vanden Berghe T, Linkermann A, Jouan-Lanhouet S, Walczak H and Vandenabeele P. Regulated necrosis: the expanding network of non-apoptotic celldeath pathways. Nat Rev Mol Cell Biol 2014; 15: 135-147.

[11] Manolis Pasparakis and Peter Vandenabeele. Necroptosis and its role in inflammation. Nature 2015; 517: 311-320

[12] Alexei Degterev, Zhihong Huang, Michael Boyce, Yaqiao Li, Prakash Jagtap, Noboru Mizushima, Gregory D Cuny, Timothy J Mitchison, Michael A Moskowitz and Junying Yuan. Chemical inhibitor of nonapoptotic cell death with therapeutic potential for ischemic brain injury. Nat Chem Biol 2005; 1: $112-119$.

[13] Ichiseki T, Kaneuji A, Katsuda S, Ueda Y, Sugimori T and Matsumoto T. DNA oxidation injury in bone early after steroid administration is involved in the pathogenesis of steroid-induced osteonecrosis. Rheumatology (Oxford) 2005; 44: 456-460.
[14] Yamamoto $T$, Irisa $T$, Sugioka $Y$ and Sueishi $K$. Effects of pulse methylprednisolone on bone and marrow tissues: corticosteroid-inducedosteonecrosis in rabbits. Arthritis Rheum 1997; 40: 2055-2064.

[15] Degterev A and Yuan J. Expansion and evolution of cell death programmes. Nat Rev Mol Cell Biol 2008; 9: 378-390.

[16] Kawahara A, Ohsawa Y, Matsumura H, Uchiyama $Y$ and Nagata S. Caspase-independent cell killing by Fas-associated protein with death domain. J Cell Biol 1998; 143: 1353-1360.

[17] Vandenabeele P, Galluzzi L, Vanden Berghe T and Kroemer G. Molecular mechanisms of necroptosis: an ordered cellular explosion. Nat Rev Mol Cell Biol 2010; 11: 700-714.

[18] Holler N, Zaru R, Micheau O, Thome M, Attinger A, Valitutti S, Bodmer J, Schneider P, Seed B and Tschopp J. Fas triggers an alternative, caspase-8-independent cell death pathway using the kinase RIP as effector molecule. Nat Immunol 2000; 1: 489-495.

[19] You Z, Savitz SI, Yang J, Degterev A, Yuan J, Cuny GD, Moskowitz MA and Whalen MJ. Necrostatin-1 reduces histopathology and improves functional outcome after controlled cortical impact in mice. Cereb Blood Flow Metab 2008; 28: 1564-1573. 